\title{
Fluorescent Nanogel Sensors for X-Ray Dosimetry
}

Li Jiang, ${ }^{1}$ Wenxiang Li, ${ }^{2}$ Jing Nie, ${ }^{1}$ Rensheng Wang, ${ }^{1, *}$ Xinjian Chen, ${ }^{3}$ Wenhui Fan, ${ }^{4,5,6,{ }^{*}}$ Liang Hu${ }^{1, *}$

1. State Key Laboratory of Radiation Medicine and Protection, Collaborative Innovation Center of Radiological Medicine of Jiangsu Higher Education Institutions, and School for Radiological and Interdisciplinary Sciences (RAD-X), Soochow University, Suzhou, China.

2. The First Affiliated Hospital, Zhejiang University School of Medicine, Zhejiang, China.

3. School of Electronics and Information Engineering, Soochow University, Suzhou, China.

4. Radiotherapy Division, Department of Oral and Maxillofacial-Head Neck Oncology, Shanghai Ninth People's Hospital, College of Stomatology, Shanghai Jiao Tong University School of Medicine, Shanghai, China.

5. National Clinical Research Center for Oral Diseases, Shanghai, China.

6. Shanghai Key Laboratory of Stomatology \& Shanghai Research Institute of Stomatology, Shanghai, China.

Corresponding authors:

Rensheng Wang,wrs16@suda.edu.cn

Wenhui Fan, 281809270@qq.com

Liang Hu, huliang@suda.edu.cn 


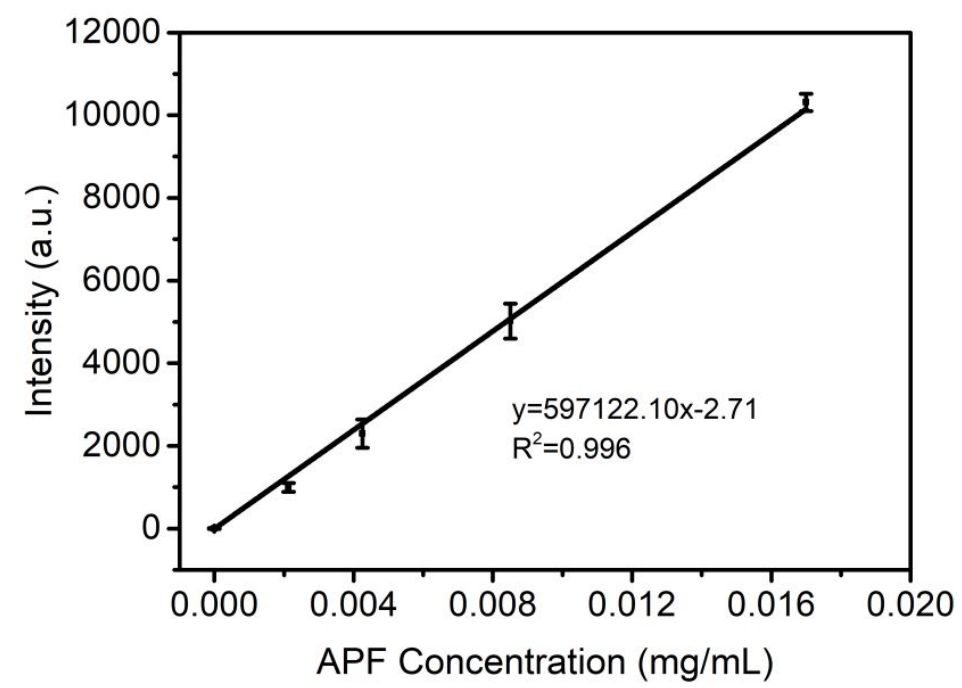

Figure S1. The quantitative correlation between APF molecule concentration and its FL intensity at $515 \mathrm{~nm}$. 

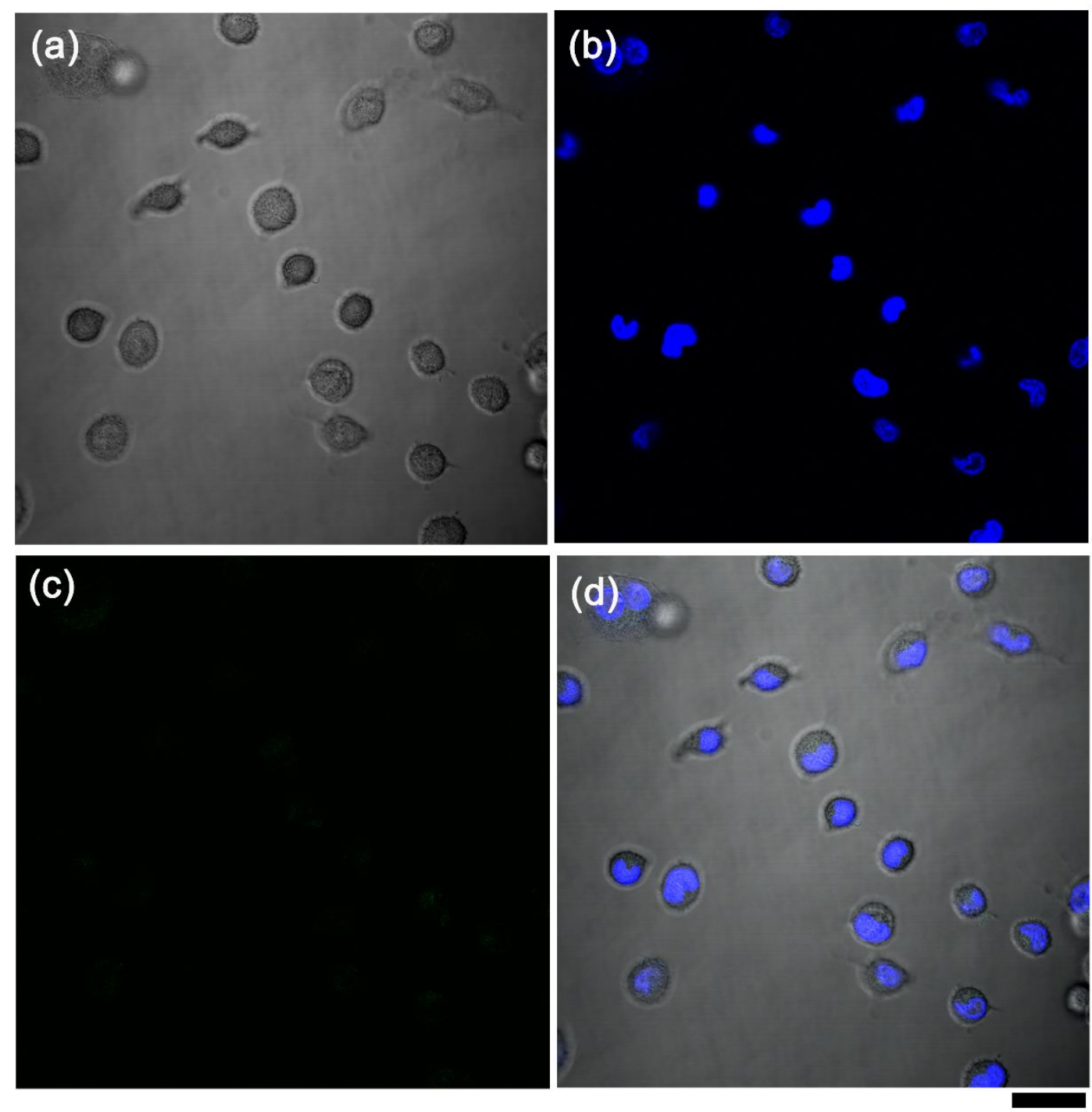

Figure S2. The confocal fluorescence images of unirradiated A549 cells. (a) Bright-field image, and images in (b) blue and (c) green channels and (d) emerged images in (a-c). The scale bar is 30 $\mu \mathrm{m}$. 

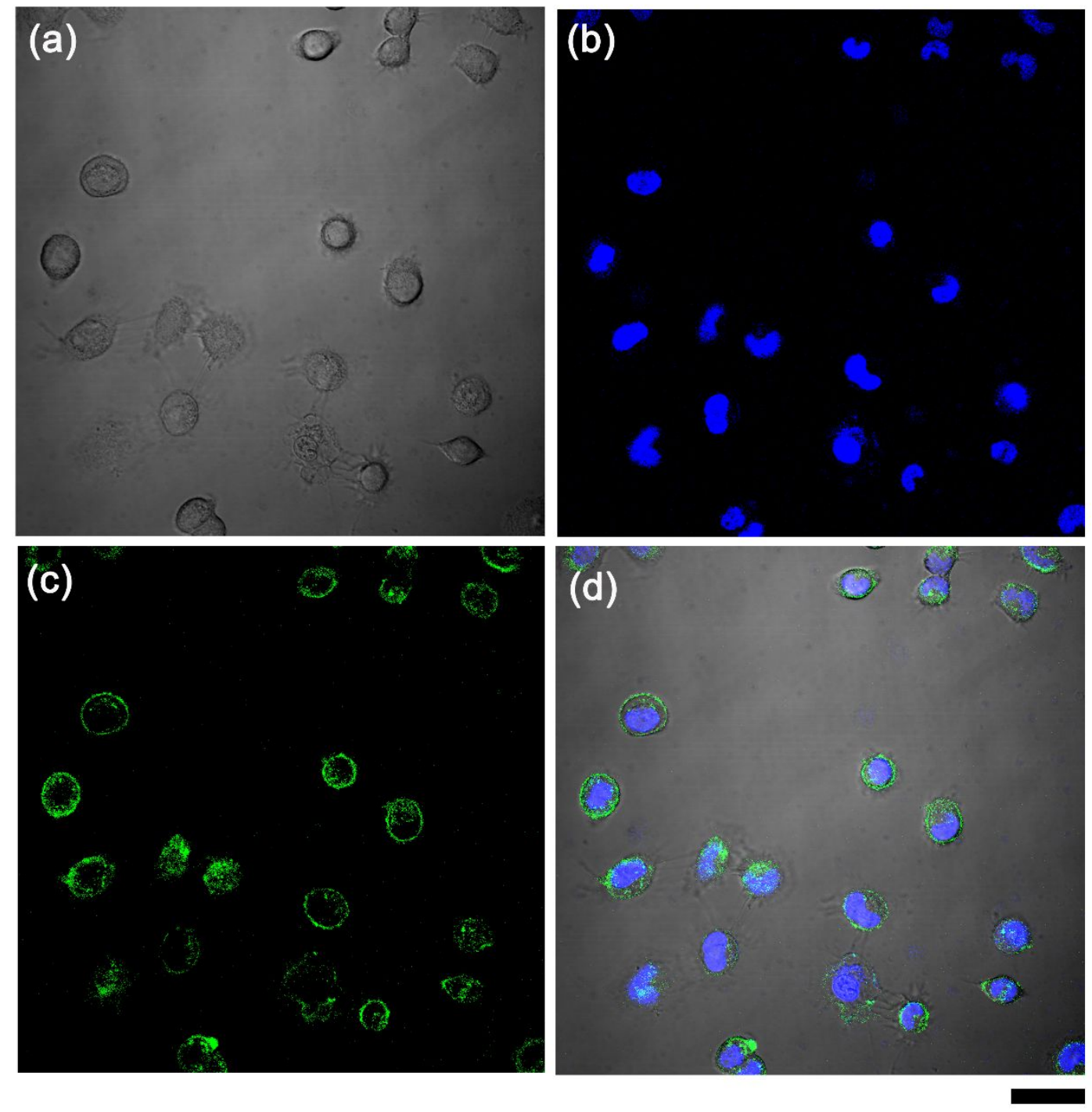

Figure S3. The confocal fluorescence images of the PAA-APF nanogel-incubated A549 cells after X-ray irradiation with 0 Gy. (a) Bright-field image, and images in (b) blue and (c) green channels and (d) emerged images in (a-c). The scale bar is $30 \mu \mathrm{m}$. 


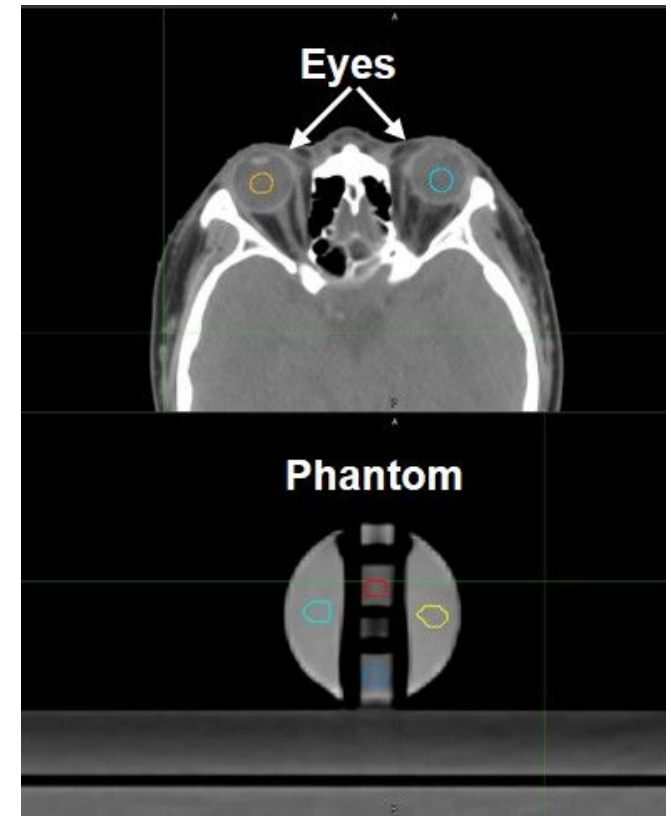

Figure S4. (Up) The CT value of the eye averaged over the circle regions was 15 HU. (Bottom) The $\mathrm{CT}$ value of the phantom averaged over the polygonal regions was $26 \mathrm{HU}$. 


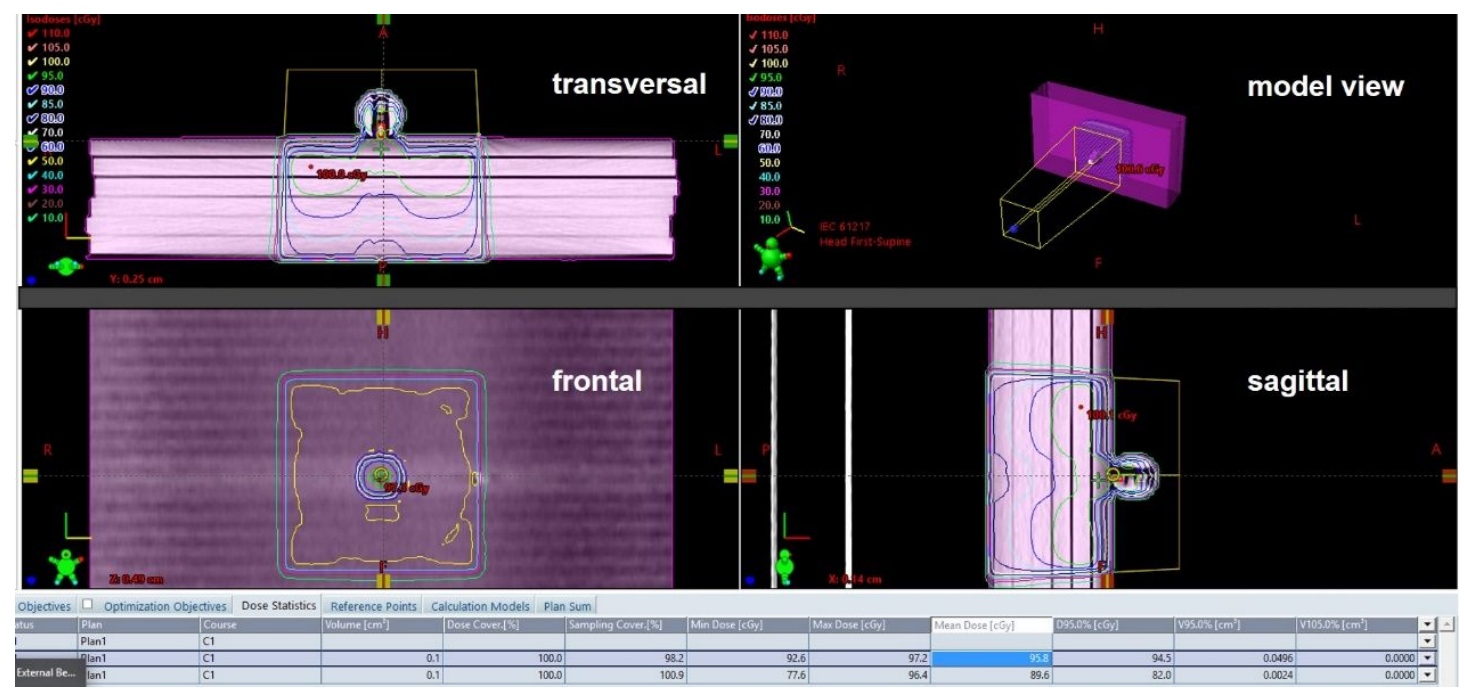

Figure S5. Doses detection at different positions in the phantom by the TPS. 
Table S1. The properties of gel-based dosimeters

\begin{tabular}{lcccc}
\hline Main chemical composition & LOD & Linear range & Waiting time & Measuring method \\
\hline Acrylamide, gold nanoparticles[1] & $10 \mathrm{~Gy}$ & $0-25 \mathrm{~Gy}$ & $>24 \mathrm{~h}$ & MRI \\
Xylenol Orange, gelatin[2] & $5 \mathrm{~Gy}$ & $5-30 \mathrm{~Gy}$ & $12 \mathrm{~h}$ & Spectrophotometry \\
Acrylamide[3] & $2 \mathrm{~Gy}$ & $0-20 \mathrm{~Gy}$ & $4 \mathrm{~h}$ & CT \\
xylenol orange, PVA[4] & $1 \mathrm{~Gy}$ & $0-30 \mathrm{~Gy}$ & 30 min & Spectrophotometry \\
HAuCl & & & & Absorbance \\
This agarose[5] & $0.5 \mathrm{~Gy}$ & $0-3 \mathrm{~Gy}$ & $1 \mathrm{~h}$ & spectroscopy \\
\hline
\end{tabular}

\section{Reference}

[1] W.N. Rahman, C.J. Wong, T. Ackerly, N. Yagi, M. Geso, Polymer gels impregnated with gold nanoparticles implemented for measurements of radiation dose enhancement in synchrotron and conventional radiotherapy type beams, Australas Phys Eng Sci Med 35 (2012) 301-309.

[2] G. Magugliani, G.M. Liosi, S. Andreoli, S. De Crescenzo, E. Mossini, E. Macerata, M. Mariani, Characterization of Fricke-gelatin dosimeters for intraoperative Radiation Therapy dosimetry, Radiation Physics and Chemistry 168 (2020) 108629.

[3] C. Matrosic, W. Culberson, B. Rosen, E. Madsen, G. Frank, B. Bednarz, Initial characterization of a gel patch dosimeter for in vivo dosimetry, Physics in Medicine and Biology 61 (2016) N240-N248.

[4] S.T. Smith, K.-S. Masters, K. Hosokawa, J.P. Blinco, S.B. Crowe, T. Kairn, J.V. Trapp, Technical Note: Preliminary investigations into the use of a functionalised polymer to reduce diffusion in Fricke gel dosimeters, Am. Assoc. Phys. Med. 42 (2015) 6798-6803. 
[5] K. Pushpavanam, S. Inamdar, J. Chang, T. Bista, S. Sapareto, K. Rege, Detection of Therapeutic Levels of lonizing Radiation Using Plasmonic Nanosensor Gels, Advanced Functional Materials 27 (2017) 1606724. 
Table S2 Comparison elemental analysis of the eye phantom and human eyes.

\begin{tabular}{|c|c|c|c|c|}
\hline & C(wt.\%) & H(wt.\%) & O(wt.\%) & N(wt.\%) \\
\hline eye phantom ${ }^{1}$ & 68.76 & 8.68 & 19.99 & 3.03 \\
\hline human eyes ${ }^{2}$ & 11.96 & 0.5 & 83.47 & 4.08 \\
\hline
\end{tabular}

1. The elemental content $(\mathrm{C}, \mathrm{H}, \mathrm{O}, \mathrm{N})$ was determined with an elemental analyzer (Vario ELCube).

2. Data was obtained from Geant 4 User's Guide for Application Developers, 2014. 